\title{
What is the utility of using syndromic surveillance systems during large subnational infectious gastrointestinal disease outbreaks? An observational study using case studies from the past 5 years in England
}

\author{
D. TODKILL ${ }^{1,2 *}$, A. J. ELLIOT ${ }^{1}$, R. MORBEY ${ }^{1}$, J. HARRIS $^{3}$, J. HAWKER $^{2}$, \\ O. EDEGHERE ${ }^{2}$ AND G. E. SMITH ${ }^{1}$ \\ ${ }^{1}$ Public Health England, Real-time Syndromic Surveillance Team, National Infection Service, Birmingham, UK \\ ${ }^{2}$ Public Health England, Field Epidemiology Service, National Infection Service, Birmingham, UK \\ ${ }^{3}$ Public Health England, Gastrointestinal Emerging and Zoonotic Infections, National Infection Service, \\ Colindale, London, UK
}

Received 27 July 2015; Final revision 10 February 2016; Accepted 19 February 2016; first published online 1 April 2016

\section{SUMMARY}

Syndromic surveillance systems in England have demonstrated utility in the early identification of seasonal gastrointestinal illness (GI) tracking its spatio-temporal distribution and enabling early public health action. There would be additional public health utility if syndromic surveillance systems could detect or track subnational infectious disease outbreaks. To investigate using syndromic surveillance for this purpose we retrospectively identified eight large GI outbreaks between 2009 and 2014 (four randomly and four purposively sampled). We then examined syndromic surveillance information prospectively collected by the Real-time Syndromic Surveillance team within Public Health England for evidence of possible outbreak-related changes. None of the outbreaks were identified contemporaneously and no alerts were made to relevant public health teams. Retrospectively, two of the outbreaks - which happened at similar times and in proximal geographical locations - demonstrated changes in the local trends of relevant syndromic indicators and exhibited a clustering of statistical alarms, but did not warrant alerting local health protection teams. Our suite of syndromic surveillance systems may be more suited to their original purposes than as means of detecting or monitoring localized, subnational GI outbreaks. This should, however, be considered in the context of this study's limitations; further prospective work is needed to fully explore the use of syndromic surveillance for this purpose. Provided geographical coverage is sufficient, syndromic surveillance systems could be able to provide reassurance of no or minor excess healthcare systems usage during localized GI incidents.

Key words: Gastrointestinal infections, outbreaks, surveillance, surveillance system.

\section{INTRODUCTION}

Syndromic surveillance is the real-time (or near real-time) collection, analysis, interpretation and dissemination of health-related data to enable the early identification of the impact (or absence of impact) of

\footnotetext{
* Author for correspondence: Dr D. Todkill, 6th Floor, 5 St Philips Place, Birmingham B3 2PW, UK.

(Email: danieltodkill@hotmail.com)
}

potential human or veterinary public health threats which require effective public health action [1].

Syndromic surveillance systems are increasingly used to identify potential human or veterinary public health threats earlier than traditional methods [2] (e.g. clinical or laboratory notification of diseases) enabling timely public health action and planning, as well as providing an assessment of increasing disease activity, for example identifying rises in seasonal norovirus [3] or monitoring pandemic influenza [4]. In 
addition, a key role of such systems is providing reassurance to decision makers during incidents or mass gathering events that there is no associated morbidity in the community. There is a growing evidence base for this role, for example during incidents such as the Eyjafjallajokull volcano eruption [5], extreme weather events [6], or the London 2012 Olympic and Paralympic Games [7]. Syndromic surveillance systems have known utility in both the early identification of changing seasonal trends of infectious gastrointestinal illness (GI) [8] and in tracking its spatial and temporal distribution at the national level [3].

The utility of syndromic surveillance in identifying or monitoring subnational infectious GI outbreaks is less clear. This has been explored in the literature previously [9-12] using either modelled or retrospectively identified case studies, with varying results. In England, a study using a synthesized cryptosporidiosis outbreak, found that the surveillance system would be unlikely to detect such an outbreak [12]. In contrast, a study using a real cryptosporidiosis outbreak in the UK demonstrated the potential to not only detect outbreaks, but follow their size, spread and tempo in near to real time [13]. This study aimed to assess whether the existing English syndromic surveillance systems are reliably able to detect large, subnational infectious gastrointestinal outbreaks in order to determine their utility in such events and inform current syndromic surveillance practices and future developments.

\section{METHODS}

\section{Syndromic surveillance systems}

In England, Public Health England (PHE) coordinates a number of real-time syndromic surveillance systems. These include a sentinel Emergency Department Syndromic Surveillance System (EDSSS) [14], General Practitioner (GP) surveillance systems that monitor 'in-hours' (GPIH) and 'out-of-hours' (GPOOH) GP consultations. The GPOOH system captures information from GP consultations during hours outside of usual surgery times, which would expect to capture information from the more severe end of the disease spectrum where cases would not wait for a regular GP appointment. The emergency department (ED) system would also capture information from more unwell or urgent cases. This is, however, a sentinel system originally developed in preparation for the 2012 London Olympics, with the majority of EDs in London. The EDSSS was included in this study if a participating
ED was present in the region of interest. A telephone health advice service (NHS 111) syndromic surveillance system is also operated and these systems have been described in detail elsewhere [15].

The PHE Real-time Syndromic Surveillance Team (ReSST) employ a statistical algorithm [16] which is executed at local authority (LA; a government administrative level in England), regional and national levels for a variety of syndromes automatically to generate statistical 'alarms' (when there is a statistically significant increase in the value of an indicator above expected values). The statistical algorithm runs independently on each system and alarms generated are recorded and reviewed contemporaneously by members of ReSST. The alarms serve to highlight a need to risk-assess data from that geographical area or syndrome. These reviews utilize a standard risk assessment process to determine whether these alarms require further monitoring or alerting of local or relevant health protection teams. Risk assessment is based on a number of factors; the size of an excess over baseline levels, comparison with national trends, if affecting multiple syndromic systems, severity and unusual spatial or age clustering (G. Smith, personal communication).

\section{Gastrointestinal outbreaks}

We compiled a sampling frame of eligible infectious GI outbreaks from those recorded on either the PHEmaintained Electronic Foodborne or Non-Foodborne Gastrointestinal Outbreak Surveillance System (eFOSS) database [17], Health Protection Zone (HP Zone) Dashboard [18] data management system or the weekly PHE Health Protection Bulletin [19].

Eligible outbreaks were large GI outbreaks involving $>75$ symptomatic cases, suspected to be caused by a viral, bacterial or protozoan organism commonly causing GI symptoms such as diarrhoea, nausea or vomiting and that occurred during 1 May 2009 to 1 May 2014. A 5-year period was chosen due to the relative rarity of subnational outbreaks of this magnitude. A simple random sample of four outbreaks was selected from the sampling frame with a further four outbreaks purposively selected based on localized geography, size of the outbreak ( $>75$ cases) and a short duration ( $\leqslant 3$ weeks); factors which we hypothesized would make outbreaks more likely to be detected by syndromic surveillance and not on the coverage or operation of syndromic surveillance systems at the time. A review of determinants of outbreak detection through automated surveillance demonstrated 
magnitude and shape of a signal and timing as important determinants [20].

We obtained written outbreaks reports and contacted local PHE Health Protection teams to gather information on the geographical location of selected outbreaks, and the duration of each outbreak based on the date of likely exposure (or date of first reported case) and date of last reported case, or if no putative exposure was found, from the time of first reported cases to last reported case.

\section{Data analysis}

We assessed the ability of each syndromic surveillance system to detect each outbreak by visually examining time-series graphs of the following syndromic surveillance indicators: 'vomiting', 'diarrhoea' and 'gastroenteritis' at the LA or former primary care trust (PCT) area, regional and national geographical levels. For datasets collected prior to August 2013, 'regional' refers to one of ten Strategic Health Authorities (SHA) in England [former administrative organizations of the National Health Service (NHS)], and after August 2013 it refers to one of 15 PHE centres, the local presence of PHE. This change in denominator will not have adversely affected results; the change did not happen during the period of the outbreaks or comparator time periods.

The time-series graphs plotted data 1 month on either side of the outbreak period for the relevant year as well as the same time period in the preceding and following year (if available) as comparators.

All statistical alarms and corresponding alerts sent to relevant health protection teams at the time of the selected GI outbreaks were reviewed to determine whether these were related to the outbreak. A comparison between alarms made in comparator years was not made due to changes in statistical methods. This change in statistical process would not have impacted the results of this study; this study was retrospective and review of data was conducted regardless of presence or absence of alarms. It is, however, possible that these statistical processes may have been influenced if health protection teams were alerted at the time of the outbreak, as the data review and risk assessment undertaken at that geography may or may not have happened based soley on alarm activity.

\section{RESULTS}

During the study period, a total of 101 eligible GI outbreaks were reported across England. The characteristics of the eight outbreaks included in this study are summarized in Table 1 .

During all the selected outbreaks, no public health action had been taken based upon syndromic surveillance data to highlight possible GI-related incidents to relevant health protection teams. During the outbreak periods identified, the telephone health advice service (NHS 111) system did not have data available, due to either transit between service providers or system downtime to enable system improvements to be made. A summary of the key findings is provided in Table 2, and in Table 3 the syndromic surveillance systems which were either in operation, or for EDSSS, included if a sentinel ED was present in the geographical region.

Outbreaks 1-4 were purposively selected, and outbreaks 5-8 selected at random.

\section{Outbreaks 1 and 2: Outbreaks of Salmonella species following a university ball and street food festival, February-March 2013}

Outbreaks 1 and 2 were two separate, distinct outbreaks with overlapping dates of exposure that occurred within the North East (NE) PHE centre geographical region, although in two different cities (Newcastle upon Tyne and Durham). As cases may have accessed healthcare services in neighbouring areas, and some syndromic systems provide granularity to PHE region only, the individual outbreaks were not considered in isolation.

\section{Description of syndromic surveillance systems' data}

Data from the GPIH and GPOOH systems were analysed for this outbreak (no EDs in the NE were participating in the EDSSS at the time).

There was an increase in the daily GP consultation rate (GPIH) for GI-related conditions midweek (6 March 2013), both in the NE region and nationally (but not in other individual regions). During the outbreak periods, we observed the highest GP consultation rates for gastroenteritis and diarrhoea compared to the preceding and following months, and comparator years. At the local authority level, there was greater variability in daily GP consultation rates for similar conditions but in Newcastle LA an increase in gastroenteritis was observed from 4 to 8 March, which was higher than the preceding or following month; similar patterns were observed in neighbouring North Tyneside, County Durham and Gateshead LAs (Fig. 1). 
Table 1. Selected characteristics of gastrointestinal outbreaks included in the study

\begin{tabular}{|c|c|c|c|c|c|}
\hline $\begin{array}{l}\text { Outbreak no. } \\
\text { and reference }\end{array}$ & Organism & $\begin{array}{l}\text { No. of possible, } \\
\text { probable and } \\
\text { confirmed cases } \\
\text { reported }\end{array}$ & Setting & $\begin{array}{l}\text { Start of outbreak } \\
\text { to last reported } \\
\text { case or case } \\
\text { definition }\end{array}$ & $\begin{array}{l}\text { Geographical region(s) } \\
\text { predominantly affected }\end{array}$ \\
\hline $1^{*}$ & $\begin{array}{l}\text { Salmonella } \\
\text { DT120 }\end{array}$ & 113 & University ball & 1-5 Mar. 2013 & North East \\
\hline $2[26]$ & $\begin{array}{l}\text { Salmonella } \\
\text { Agona PT40 }\end{array}$ & 592 & $\begin{array}{l}\text { 3-day street food } \\
\text { festival }\end{array}$ & $\begin{array}{l}28 \text { Feb. to } 7 \text { Mar. } \\
2013\end{array}$ & North East \\
\hline $3[27]$ & Not identified & $\begin{array}{l}338 \text { (152 in London } \\
\text { region, } 68 \text { in Kent, } \\
\text { Surrey and Sussex) }\end{array}$ & $\begin{array}{l}\text { Organized swimming } \\
\text { competition in the } \\
\text { Thames river }\end{array}$ & 7-16 Oct. 2012 & London, South East \\
\hline $4^{*}$ & $\begin{array}{l}\text { Norovirus } \\
\text { (suspected) }\end{array}$ & 457 & Secondary school & 17-19 Dec. 2012 & South East \\
\hline $5^{*}$ & Bacillus cereus & 230 & $\begin{array}{l}\text { Nurseries } \\
\text { geographically } \\
\text { dispersed across the } \\
\text { South East }\end{array}$ & $\begin{array}{l}30 \text { May to } 1 \text { June } \\
2012\end{array}$ & South East \\
\hline $6[28]$ & $\begin{array}{l}\text { Clostridium } \\
\text { perfringens }\end{array}$ & 150 & Secondary school & 21-23 Mar. 2013 & London \\
\hline $7^{*}$ & Campylobacter & 89 & $\begin{array}{l}\text { Residential educational } \\
\text { institute }\end{array}$ & $\begin{array}{l}18 \text { May } 2010 \text { to } \\
6 \text { June } 2010\end{array}$ & Yorkshire \& Humber \\
\hline 8 [29] & $\begin{array}{l}\text { Salmonella } \\
\text { enterica }\end{array}$ & 327 & Prison & 13-20 Sept. 2009 & London \\
\hline
\end{tabular}

* Outbreak details gathered from personal communication with relevant health protection teams.

Table 2. Key findings

\begin{tabular}{|c|c|c|}
\hline $\begin{array}{l}\text { Outbreak } \\
\text { no. }\end{array}$ & Statistical alarms during outbreak period & $\begin{array}{l}\text { Changes in trends in syndromic surveillance systems } \\
\text { during outbreak period }\end{array}$ \\
\hline $1 / 2$ & $\begin{array}{l}\text { Statistical alarms in the North East in GPOOH for } \\
\text { 'vomiting', 'diarrhoea' at regional level. Weekly alarm } \\
\text { in GPIH for 'vomiting', 'gastroenteritis' and } \\
\text { 'diarrhoea' }\end{array}$ & $\begin{array}{l}\text { High levels of mid-week GPIH consultations for } \\
\text { GI-related conditions midweek ( } 6 \text { March) nationally, } \\
\text { in the North East region and Newcastle local authority } \\
\text { area compared to comparator periods. High level of } \\
\text { mid-week GPOOH compared to preceding and } \\
\text { following months. }\end{array}$ \\
\hline 3 & $\begin{array}{l}\text { Three national alarms; for 'vomiting' in GPOOH and } \\
\text { EDSSS and diarrhoea in GPIH surveillance system. } \\
\text { Alarms in affected regions; three 'diarrhoea' in GPIH } \\
\text { and one in GPOOH for 'gastroenteritis'. Weekly } \\
\text { alarms in affected local health authority in GPIH for } \\
\text { 'vomiting' and 'diarrhoea' }\end{array}$ & Appear consistent with comparator time periods \\
\hline 4 & $\begin{array}{l}\text { Weekly alarms for GPIH surveillance system at local } \\
\text { health authority level for 'gastroenteritis' and } \\
\text { 'vomiting' }\end{array}$ & Appear consistent with comparator time periods \\
\hline 5 & No relevant alarms & Appear consistent with comparator time periods \\
\hline 6 & No relevant alarms & $\begin{array}{l}\text { Non-statistically significant mid-week rise in contacts to } \\
\text { GPOOH in affected local authority region }\end{array}$ \\
\hline 7 & No relevant alarms & Appear consistent with comparator time periods \\
\hline 8 & No relevant alarms & Appear consistent with comparator time periods \\
\hline
\end{tabular}

GPOOH, General Practitioner 'out-of-hours' consultations; GPIH, General Practitioner 'in-hours' consultations; GI, gastrointestinal illness; EDSSS, Emergency Department Syndromic Surveillance System. 
Table 3. Syndromic surveillance systems in operation or for EDSSS, included if a sentinel emergency department was present in the geographical region

\begin{tabular}{ll}
\hline \hline Outbreak no. & Syndromic surveillance system evaluated \\
\hline $1 / 2$ & GPIH, GPOOH \\
3 & GPIH, GPOOH, EDSSS \\
4 & GPIH, GPOOH, EDSSS \\
5 & GPIH, GPOOH, EDSSS \\
6 & GPIH, GPOOH, EDSSS \\
7 & Weekly GPIH \\
8 & Weekly GPIH \\
\hline \hline
\end{tabular}

GPIH, General Practitioner 'in-hours' consultations; GPOOH, General Practitioner 'out-of-hours' consultations; EDSSS, Emergency Department Syndromic Surveillance System.

The highest proportion of GI-related consultations to GPOOH providers in the NE region, compared to the preceding and following months, was recorded mid-week on Wednesday 6 March 2013.

\section{Statistical alarms}

Three statistical alarms were generated in the NE in the GPOOH system during the outbreak period. These included regional alarms on 2 March 2013 for 'vomiting' and on 3 March for 'diarrhoea'. The third alarm was at the LA level for weekly GPIH consultations for 'vomiting', 'gastroenteritis' and 'diarrhoea requiring oral rehydration' in the North Tyneside PCT area for the week commencing 4 March. At the time, ReSST continued to monitor, and the risk assessment did not warrant alerting local health protection teams.

\section{Outbreak 3: Outbreak of diarrhoea and vomiting following an organized river swim, October 2012}

\section{Description of syndromic surveillance systems' data}

For all syndromic indicators, the pattern and rates of GP consultations (GPIH and GPOOH systems) and ED attendances (EDSSS) during the outbreak period were similar to those observed during the comparator time periods at national, regional and local levels.

\section{Statistical alarms}

During the outbreak period there were three national alarms for 'vomiting' in the GPOOH system on 10 October, 'diarrhoea' in the GPIH system on 12 October and 'vomiting' in the EDSSS on 13 October.
The outbreak resulted in $>50$ reported cases each in two regions [London and South East (SE) coast], both of which had statistical alarms during the outbreak period. The GPIH system alarmed for 'diarrhoea' consultations in the SE coast area on 8 October and 12 October, and in London on 12 October. The GPOOH system alarmed for gastroenteritis consultations on 13 October in the SE. Weekly alarms for GP consultations for 'vomiting' and 'diarrhoea' also occurred in the GPIH system in a local area (Richmond and Twickenham PCT). The risk assessment undertaken at the time of these alarms classified them as low risk and no alerts were issued to local health protection teams.

\section{Outbreak 4: Outbreak of norovirus at a high school, December 2012}

Description of syndromic surveillance systems' data

Rates of contacts to GPIH, GPOOH and EDSSS were consistent with the comparator time periods at local, regional and national levels.

\section{Statistical alarms}

No relevant statistical alarms were generated at national or regional level during the outbreak. Weekly alarms for GPIH at the local PCT level in the West Sussex area were generated during the week of the outbreak for both 'gastroenteritis' and 'diarrhoea'. This is, however, in the context of multiple (four) statistical alarms for 'gastroenteritis' during the preceding and following months for that geographical area.

\section{Outbreak 5: Outbreak of Bacillus cereus in multiple nurseries, May-June 2012}

Description of syndromic surveillance systems' data

The trends in GI syndromic indicators observed in the outbreak period at local, regional and national levels were consistent with the comparator period across the GPOOH, GPIH and EDSSS.

\section{Statistical alarms}

There were no relevant statistical alarms during, or close to the period of the outbreak.

\section{Outbreak 6: Outbreak of Clostridium perfringens in a secondary school, March 2013}

Description of syndromic surveillance systems' data

There was a non-statistically significant rise in midweek contacts to GPOOH in Richmond upon 


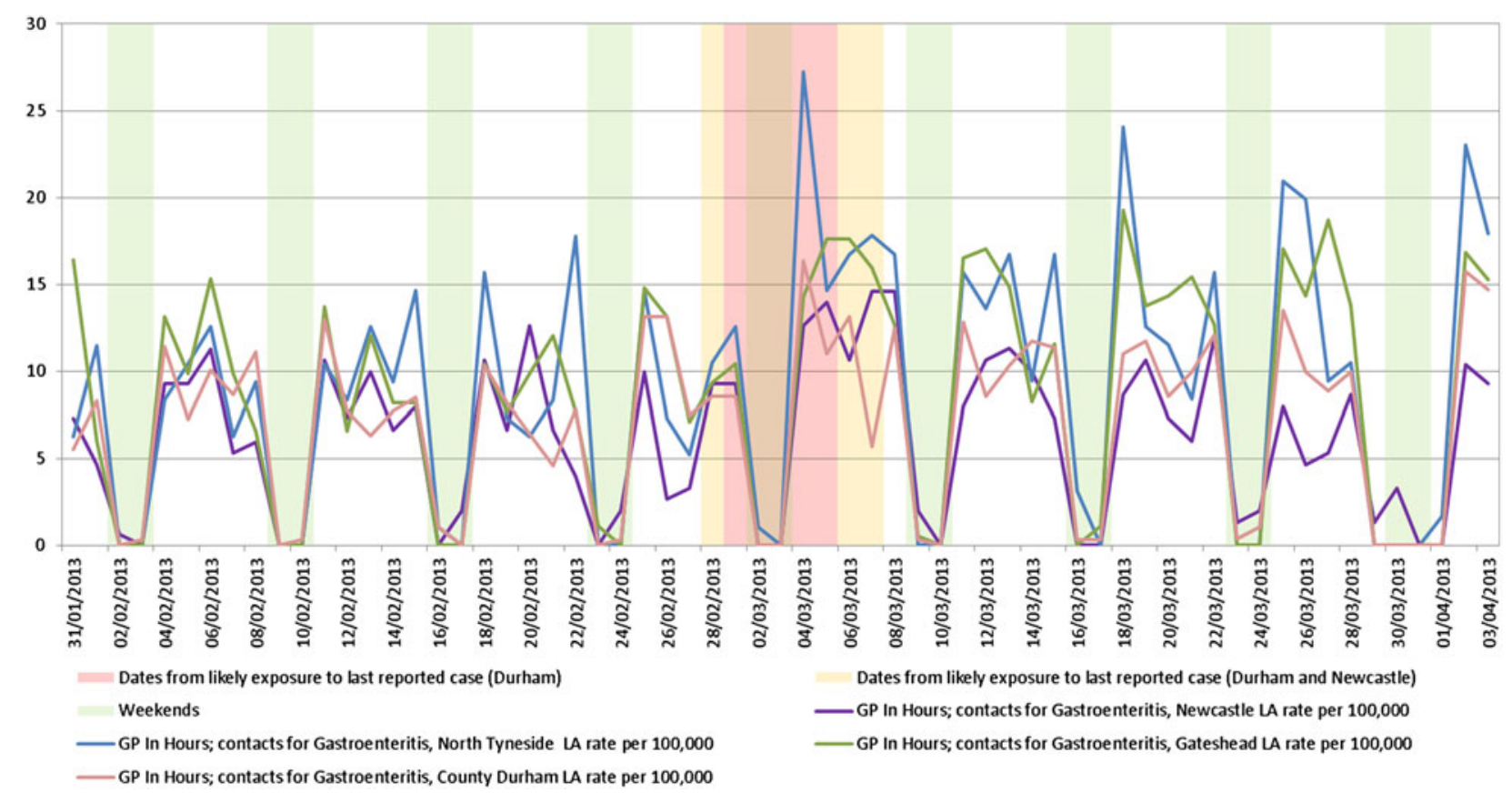

Fig. 1. Rates per 100000 of General Practitioner 'in-hours' surveillance system contacts for gastroenteritis, North East local authority areas, 31 January 2013 to 3 April 2013

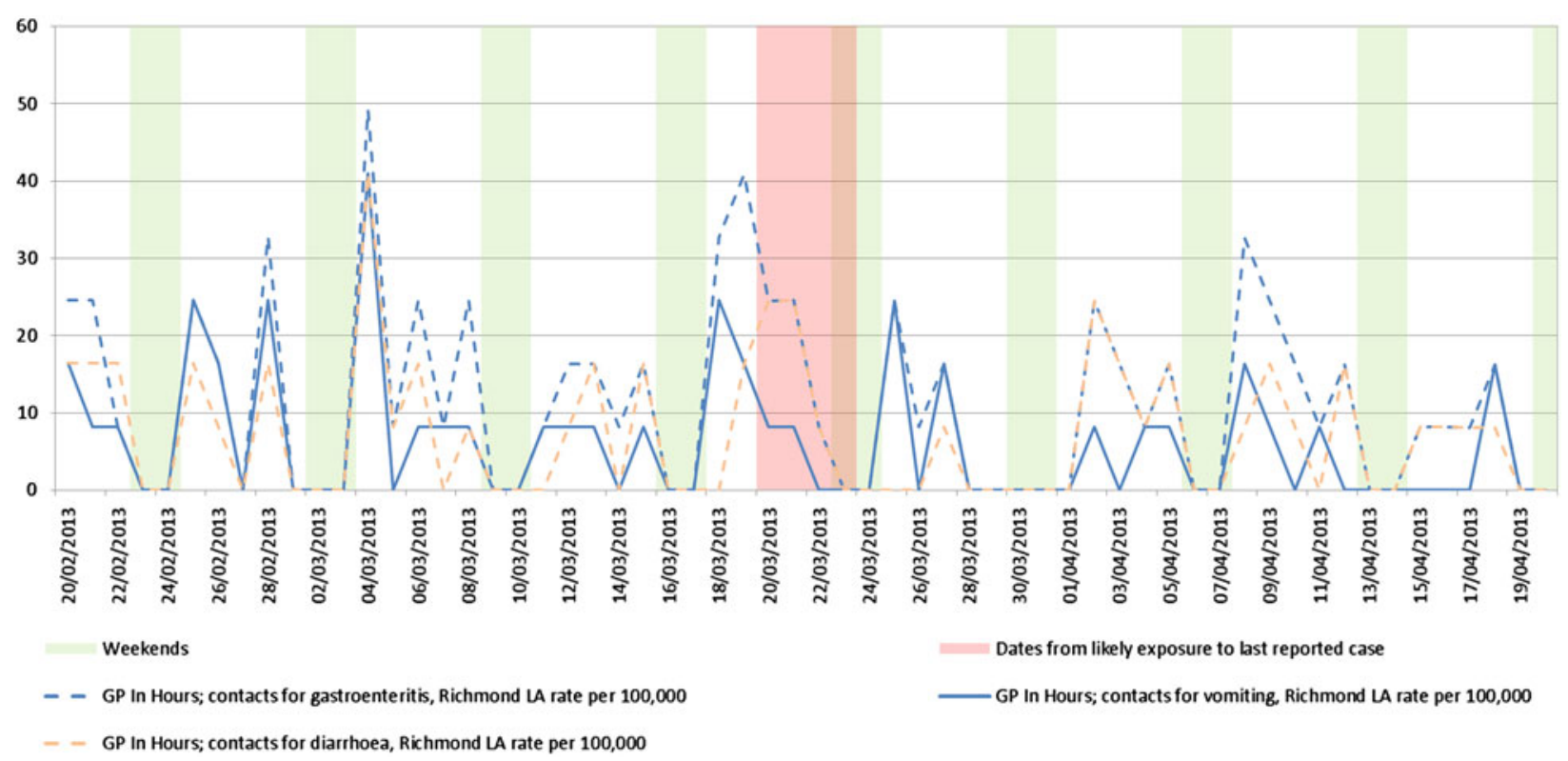

Fig. 2. Rates per 100000 of General Practitioner 'in-hours' surveillance system contacts for gastroenteritis, vomiting and diarrhoea in Richmond upon Thames local authority, 20 February 2013 to 9 April 2013.

Thames LA geographical area during the outbreak (Fig. 2). No differences between the comparator periods and the outbreak period could be observed at regional or national levels for the GPOOH and GPIH systems.
During the period of the outbreak, there were no changes in the pattern of GI-related contacts in the GPOOH or EDSSS in the London regional area compared to either the preceding or following months or the previous year. 


\section{Statistical alarms}

There were no relevant alarms during, or close to the period of the outbreak.

\section{Outbreaks 7 and 8: Outbreaks of gastroenteritis at an educational institute (May-June 2010) and Salmonella in a prison (September 2009)}

Description of syndromic surveillance systems' data

During both these outbreaks only data from the weekly GPIH system was available and no changes in the normal pattern were identified.

\section{Statistical alarms}

No relevant alarms were detected during, or close to the period of the outbreak.

\section{DISCUSSION}

We retrospectively assessed the utility of English syndromic surveillance systems in detecting selected subnational infectious GI outbreaks that occurred during 2009-2013. To date, the focus for these surveillance systems in England has primarily been to monitor national seasonal GI activity, including annual norovirus and rotavirus epidemics [21, 22].

We found that the syndromic surveillance systems did not detect these outbreaks contemporaneously as part of routine syndromic surveillance activity undertaken at the time. Our retrospective analysis showed that for two of the outbreaks, which happened at similar times and in proximal geographical locations, there were demonstrable changes in trends for relevant syndromic indicators at the subnational level in one or more syndromic surveillance system, and a clustering of statistical alarms. However, at the time, these statistical alarms (considered among other daily alarms generated as part of the routine analyses) were assessed as low public health risk and no further action(s) was taken and the findings were not alerted to PHE local health protection teams.

Automated statistical algorithms highlight potential public health problems to the surveillance team and expedite public health risk assessment of the data. In all the outbreaks, these statistical alarms were not particularly unusual among the other alarms which occur each day and the decision not to issue an alert following a risk assessment was warranted given the context. The most unusual feature in the outbreaks was the mid-week spike in GPIH consultations for GI-related conditions during outbreak 2 on 6 March which was unusual because GPOOH 'spikes' usually occur at the weekend (when most in-hours GP practices are closed). This did not generate a statistical alarm and importantly it occurred 2 days after the food festival outbreak had already been bought to the attention of local health protection teams.

Our study shows that none of the other outbreaks evaluated could have reliably been identified by the syndromic surveillance systems that existed at the time either contemporaneously or retrospectively. These findings are consistent with those from a recent paper by Ziemann et al. [11] who demonstrated a syndromic surveillance system encompassing emergency dispatch, ambulance and ED data from 12 European countries (the UK was not included) successfully identified only one in 147 outbreaks. Other studies using ED syndromic surveillance systems [23] and ambulatory care data [24] have failed to identify localized outbreaks of GI illness. Syndromic surveillance systems using tele-health data appear to have more potential for identifying GI outbreaks at an early stage $[13,25]$.

The selection of outbreaks for this study was based on outbreak characteristics, not on proximity, coverage or operation of syndromic surveillance systems at that time or geographical area; as this study presents a pragmatic assessment rather than idealized circumstances which could have been done using modelled data. This 'real-world' assessment is also reflected in the variation in systems which were in operation at the times of the outbreaks; from the nature of syndromic surveillance which relies on passive collection of data from providers, unexpected changes to provider systems can leave surveillance systems without adequate data for operation. Moreover, coverage varies; in particular, in the UK, the ED system is sentinel and would be unlikely to detect subnational, localized outbreaks if no local EDs are participating.

We suggest that the inability of our systems to detect outbreaks despite their large size may be due to an insufficient number of persons presenting to the healthcare services monitored by our systems to trigger an alarm either because the illness was mild or self-limiting or differential health-seeking behaviour; and the population coverage of our systems at the time was insufficient in those areas affected by the outbreak. We also explored a range of diseases; disease type may influence the ability of systems to detect outbreaks; for example norovirus is typically self-limiting and patients may have different healthcare-seeking 
behaviours compared to other, more severe types of GI illness.

Outbreaks of the size we selected for are fortunately rare events in the UK, and for the purposively selected outbreaks finding 'ideal' outbreaks for the four purposively sampled was challenging; larger outbreaks may more frequently have characteristics such as cases which are dispersed over time and place, which would be less suitable for syndromic surveillance to identify.

Our study has highlighted some of the key challenges in interpreting syndromic surveillance data, particularly at local level. On the graphical review of the data, in the NE outbreaks, changes were observed at local level. Localized and more granular data are usually characterized by increased variability in daily consultations/attendances at health services and this makes the identification of real events among background 'noise' challenging, even when statistical alarms have been generated. Challenges for epidemiologists running these systems include difficulties in selecting the optimal configuration of statistical algorithms, establishing and refining baselines to improve exceedance calculations and subsequently integrating statistical alarms with epidemiological assessment of untoward trends. Whether a system is better suited to identifying gradual changes in disease incidence, continuous or point-source outbreaks may, to a degree, depend on the configuration of algorithms used.

The suite of syndromic surveillance systems operated by PHE were not developed to detect or monitor localized outbreaks of infectious disease and our study showed that the current systems were not useful in detecting or monitoring these eight outbreaks that occurred during 2009-2013. However, these findings must be considered in the context of certain study limitations. First, we assessed the systems' outbreak detection capability using a small selection of eligible outbreaks, two of which - although when combined represent a very large number of symptomatic cases were geographically indeterminable and three of which were institution based which may be systematically different to solely community-based outbreaks. For example our ability to detect an outbreak in an institution such as a prison will be dependent on whether the institution is served by one GP practice which does or does not contribute to the surveillance system. Second, the population coverage of the suite of syndromic surveillance systems increased over the study period and continues to expand. This suggests that the performance (sensitivity) of the current systems may be considerably better than the system that existed at the time of the outbreaks due to increased case ascertainment. Third, the NHS telehealth service (NHS 111) was not in operation during the identified outbreaks, which was potentially the most promising of the systems at identifying this type of outbreak.

\section{CONCLUSIONS AND RECOMMENDATIONS}

The small number of subnational gastrointestinal outbreaks selected for this study were not contemporaneously detected by syndromic surveillance. Retrospectively, and following particular scrutiny of the data, only two outbreaks (which happened at the same time and were geographically proximal) appear to have been picked up through a series of statistical alarms during the period. Based on our findings, the systems that existed at the time of these outbreaks were more suited to their original purpose of monitoring seasonal trends at national or regional levels than detecting or monitoring local GI outbreaks. However, this needs to be considered in terms of both the limitations of this study, and that this study is not an assessment of current syndromic surveillance systems, which have increased in both coverage and sophistication. Further work should involve prospectively assessing our system's ability to identify known outbreaks by identifying all outbreaks which have been identified via non-syndromic surveillance routes (as a 'gold standard') and monitoring syndromic indicators at local and regional levels to describe what (if any) features make GI outbreaks identifiable by syndromic surveillance systems and whether such systems have utility in this area. Sub-national outbreaks of this magnitude are sufficiently rare that gathering data for such a study may take a number of years. In addition, future work should address assessing the utility of the telephone health advice syndromic surveillance system in identifying sub-national outbreaks.

This work has informed the ongoing improvement of the national syndromic surveillance service. Importantly, the primary remit of syndromic surveillance does not include the detection and monitoring of local outbreaks of disease; local public health alerting and response mechanisms are in place to deal with this level of public health incident. Nonetheless, if the coverage of our systems is sufficient in a local area, during an outbreak, although it might not be able to detect 
the outbreak, reassurance could still be provided by syndromic surveillance, to the local health protection teams that there is not an excess burden on healthcare services.

\section{ACKNOWLEDGEMENTS}

The authors thank the Health Protection and Field Epidemiology Teams who provided information on the outbreaks included in this paper, and the Gastrointestinal, Emerging and Zoonotic Infections Department, Public Health England for providing information from the eFOSS database. We also acknowledge support from: Royal College of Emergency Medicine, EDs participating in the emergency department system (EDSSS), EMIS Health and L2S2 Ltd; $\mathrm{OOH}$ providers submitting data to the GPOOH surveillance system and Advanced Heath \& Care; TPP and participating SystmOne practices and the University of Nottingham, ClinRisk, EMIS and EMIS practices submitting data to the QSurveillance database.

\section{DECLARATION OF INTEREST}

None.

\section{REFERENCES}

1. Triple S Project. Assessment of syndromic surveillance in Europe. Lancet 2011; 378: 1833-1834.

2. Lawson AB, Kleinman K. Spatial and syndromic surveillance for public health (chapter 1). In Lawson AB, Kleinman K, eds. Spatial and Syndromic Surveillance for Public Health. Chichester: John Wiley \& Sons Ltd, 2005.

3. Cooper DL, et al. Tracking the spatial diffusion of influenza and norovirus using telehealth data: a spatiotemporal analysis of syndromic data. BMC Medicine 2008; 6: 16.

4. Cooper D, et al. Can syndromic thresholds provide early warning of national influenza outbreaks? Journal of Public Health 2009; 31: 17-25.

5. Elliott A, et al. Syndromic surveillance to assess the potential public health impact of the Icelandic volcanic ash plume across the United Kingdom, April 2010. Eurosurveillance 2010; 15.

6. Elliot A, et al. The impact of thunderstorm asthma on emergency department attendances across London during July 2013. Emergency Medicine Journal 2013; emermed-2013-203122.

7. Elliot A, et al. Syndromic surveillance - a public health legacy of the London 2012 Olympic and Paralympic Games. Public Health 2013; 127: 777-781.

8. Smith G, et al. Developing a national primary carebased early warning system for health protection $-\mathrm{a}$ surveillance tool for the future? Analysis of routinely collected data. Journal of Public Health 2007; 29: 75-82.

9. Edge VL, et al. Syndromic surveillance of gastrointestinal illness using pharmacy over-the-counter sales: a retrospective study of waterborne outbreaks in Saskatchewan and Ontario. Canadian Journal of Public Health 2004; 95: 446-450.

10. Straetemans M, et al. Automatic outbreak detection algorithm versus electronic reporting system. Emerging Infectious Diseases 2008; 14: 1610.

11. Ziemann A, et al. A concept for routine emergency-care data-based syndromic surveillance in Europe. Epidemiology and Infection 2014; 142: 2433-2446.

12. Cooper D, et al. Can syndromic surveillance data detect local outbreaks of communicable disease? A model using a historical cryptosporidiosis outbreak. Epidemiology and Infection 2006; 134: 13-20.

13. Smith S, et al. Value of syndromic surveillance in monitoring a focal waterborne outbreak due to an unusual Cryptosporidium genotype in Northamptonshire, United Kingdom, June-July 2008. Eurosurveillance 2010; 15: 19643.

14. Elliot AJ, et al. Establishing an emergency department syndromic surveillance system to support the London 2012 Olympic and Paralympic Games. Emergency Medicine Journal 2012; 29: 954-960.

15. Public Health England. Real Time Syndromic Surveillance Team website. (https://wwwgovuk/government/ collections/syndromic-surveillance-systems-and-analyses). Accessed 1 April 2015.

16. Morbey RA, et al. The application of a novel 'rising activity, multi-level mixed effects, indicator emphasis '(RAMMIE) method for syndromic surveillance in England. Bioinformatics 2015; 31: 3660-3665.

17. Public Health England. Electronic Foodborne and NonFoodbourne Gastrointestinal Outbreak Surveillance System (eFOSS). (https://bioinfosecurepheorguk/efoss). Accessed 27 April 2015.

18. Kara-Zaïtri C, Gelletlie R, Schweiger M. The development and deployment of a national web-based system for communicable disease control in England. International Journal of Infectious Diseases 2012; 16: e133.

19. Public Health England. Health Protection Reports (https://wwwgovuk/government/collections/health-protectionreport-latest-infection-reports). Accessed 27 April 2015.

20. Buckeridge DL. Outbreak detection through automated surveillance: a review of the determinants of detection. Journal of Biomedical Informatics 2007; 40: 370-379.

21. Loveridge $\mathbf{P}$, et al. Vomiting calls to NHS Direct provide an early warning of norovirus outbreaks in hospitals. Journal of Hospital Infection 2010; 74: 385-393.

22. Bawa $\mathbf{Z}$, et al. Assessing the likely impact of a rotavirus vaccination programme in England; the contribution of syndromic surveillance. Clinical Infectious Diseases 2015; 61: 77-85.

23. Balter $\mathbf{S}$, et al. Three years of emergency department gastrointestinal syndromic surveillance in New York City: what have we found. Morbidity and Mortality Weekly Report 2004; 54: 175-180. 
24. Yih WK, et al. Ambulatory-care diagnoses as potential indicators of outbreaks of gastrointestinal illness Minnesota. Morbidity and Mortality Weekly Report 2005; 54: 157-162.

25. Andersson T, et al. Syndromic surveillance for local outbreak detection and awareness: evaluating outbreak signals of acute gastroenteritis in telephone triage, web-based queries and over-the-counter pharmacy sales. Epidemiology and Infection 2014; 142: 303-313.

26. Foster K. Outbreak Report: Outbreak of Salmonella Agona phage type 40 associated with the Street Spice Festival, Newcastle upon Tyne February/March 2013 (https://wwwnewcastlegovuk/sites/drupalnccnewcastlegovuk/files/wwwfileroot/environment/environmental_ health/20130617_street_spice_oct_report_-_finalpdf). Accessed 1 April 2015.
27. Field Epidemiology Services PHE. Epidemiological investigation of an outbreak of gastrointestinal illness following a mass-participation swim in the River Thames London October 2012 (https://wwwgovuk/government/ uploads/system/uploads/attachment_data/file/33170/Thames Swim-epidemiological_report_on_outbreak_of_gastrointestinal_illnesspdf). Accessed 1 April 2015.

28. Simone B, et al. Investigating an outbreak of Clostridium perfringens gastroenteritis in a school using smartphone technology, London, March 2013. Eurosurveillance 2014; 19.

29. Davies A, et al. Salmonella enterica serovar Enteritidis phage type 4 outbreak associated with eggs in a large prison, London 2009: an investigation using cohort and case/non-case study methodology. Epidemiology and Infection 2013; 141: 931-940. 